\title{
Measurement Device for Stress Level and Vital Sign Based on Sensor Fusion
}

\author{
Noor Cholis Basjaruddin, Febian Syahbarudin, Ediana Sutjiredjeki \\ Department of Electrical Engineering, Politeknik Negeri Bandung, Bandung, Indonesia
}

Objectives: Medical health monitoring generally refers to two important aspects of health, namely, physical and mental health. Physical health can be measured through the basic parameters of normal values of vital signs, while mental health can be known from the prevalence of mental and emotional disorders, such as stress. Currently, the medical devices that are generally used to measure these two aspects of health are still separate, so they are less effective than they might be otherwise. To overcome this problem, we designed and realized a device that can measure stress levels through vital signs of the body, namely, heart rate, oxygen saturation, body temperature, and galvanic skin response (GSR). Methods: The sensor fusion method is used to process data from multiple sensors, so the output that shows the stress level and health status of vital signs can be more accurate and precise. Results: Based on the results of testing, this device is able to show the health status of vital signs and stress levels within \pm 20 seconds, with the accuracies of body temperature measurements, oxygen saturation, and GSR of $97.227 \%, 99.4 \%$, and 98.6\%, respectively. Conclusions: A device for the measurement of stress levels and vital signs based on sensor fusion has been successfully designed and realized in accordance with the expected functions and specifications.

Keywords: Stress, Psychological, Vital Signs, Sensor Fusion, Health Status, Internet of Things

\section{Introduction}

At present, the level of public action in conducting routine health checks tends to be lacking. Based on the results of the

Submitted: May 27, 2020

Revised: 1st, September 13, 2020; 2nd, December 1, 2020

Accepted: December 22, 2020

\section{Corresponding Author}

Noor Cholis Basjaruddin

Department of Electrical Engineering, Politeknik Negeri Bandung, Jl. Gegerkalong Hilir, Ds. Ciwaruga, Bandung 40012, Indonesia. Tel: +62-8-1720-2982, E-mail: noorcholis@polban.ac.id (https://orcid. org/0000-0003-2003-7452)

This is an Open Access article distributed under the terms of the Creative Commons Attribution Non-Commercial License (http://creativecommons.org/licenses/by$\mathrm{nc} / 4.0 /$ ) which permits unrestricted non-commercial use, distribution, and reproduction in any medium, provided the original work is properly cited.

(c) 2021 The Korean Society of Medical Informatics
Kompas Research and Development poll in October 2016, almost $97 \%$ of respondents in Indonesia felt that routine health checks were beneficial. However, only one in three respondents routinely have a comprehensive health check; most of the other respondents are reluctant to have medical tests because of the high costs and lack of time to go to the hospital. Moreover, they feel that they are still healthy; therefore, comprehensive health checks seem unnecessary to them. Checking the health of an individual here refers to two important aspects of health, namely, physical health and mental health. The medical devices that are generally used to measure these two aspects of health are still separate, so that they are considered less effective than they might be otherwise.

Physical health in a person can be measured through basic parameters of normal values of vital signs. Changes in vital signs have a very important value for the monitoring of bodily functions because these changes have meaning as an 
indication of the activity of organs in the body [1]. Development of new ways of measuring human vital signs has been pursued in several studies [2-4]. According to the studies $[2,3]$, a technique to monitor human vital signs by means of radar was developed, whereas in [4], a vital sign measurement technique using a smart device was proposed. The development of vital sign measuring devices generally involves many sensors [5]. In the study of Sun et al. [5], a vital sign measuring instrument was developed that is able to measure the pulse rate, respiratory rate, and body temperature. Vital sign measurement using wearable electronics was also researched in [6]. A wearable system for measuring vital signs equipped with an energy-harvesting module was developed.

Mental health can be assessed by measuring the level of stress or monitoring an individual's mental and emotional state. The prevalence of mental and emotional disorders as indicated by stress symptoms, such as depression and anxiety, for people aged 15 and above increased from around 14 million or $6 \%$ of the total population of Indonesia in 2013 to $9.6 \%$ in 2018 . In addition, stress can also cause health problems, such as a decrease in the body's immune response, which can lead to various diseases [7] and have a detrimental impact on physical and mental health [8]. Several studies on the development of stress detection have been carried out [9-11]. In [9], stress monitoring was conducted through biosignal measurements processed using machine learning methods. In [10], a multimodel system for stress measurement was introduced, while in [11] a stress monitor using a wrist device was developed. Stress detection research that focused on the use of sensors was carried out in [12], while research focused on computing for stress detection was carried out in [13]. Stress detection using wearable physiological sensors has also been researched $[14,15]$.

Because mental and physical health are closely related, we need a device that can assess both physical and mental health simultaneously. In this work, we developed such a device, which would be able to provide early warnings in the form of the health status of vital signs and stress levels using the sensor fusion method. In addition, this device can provide convenient health monitoring because it uses a mobile application, and the measurement data is stored automatically and in a cloud database. The device is expected to help users monitor their health and can provide early health warnings in the form of health status of vital signs and stress levels. Such early warnings could prompt users to visit a doctor to have their health checked.

\section{Methods}

\section{Sensor Fusion}

Sensor fusion is a data collection technique in which data obtained by several sensors is combined to provide comprehensive and accurate information. The information produced by fusion sensors is in some ways better than the information provided by separate sensors. In fusion sensor data retrieval, data is not directly retrieved from the sensor readings; rather, the sensor readings are processed first by other methods, such as the fuzzy logic method, the voting method, discrete Bayesian methods, neural networks, and Kalman filtering. The fusion process in decision making takes place at three levels, namely, sensors, fusion operators, and control applications [16].

\section{System Design}

The proposed device to measure stress levels and vital signs based on sensor fusion is able to measure a person's mental health (stress levels) and physical health (vital signs) simultaneously. This tool can be connected to a mobile application via the Internet to facilitate health monitoring, and measurement data are stored in a cloud database. In this tool, the vital signs measured are oxygen saturation level $\left(\mathrm{SpO}_{2}\right)$, body temperature, and heart rate. The stress level is measured by combining the galvanic skin response (GSR), body temperature, and heart rate sensor readings, which are then processed by a fuzzy logic algorithm in the microcontroller to determine the stress level. A system block diagram of the device is presented in Figure 1.

\section{1) Software design}

The software for this device is divided into two parts, namely, the software for the microcontroller and that for the application. The software for the microcontroller functions so that the device can read and process sensor data properly and determine the stress level with the sensor fusion method using a fuzzy logic algorithm. In addition, the microcontroller sends data processing results to cloud databases via the Internet.

Sensor reading using the sensor fusion method is done sequentially by determining the priority of initiating each component to be set in the program. This is done so that the readings between the sensors do not collide with each other. The software in the mobile application ensures that users can sign up and log in to the application and give the command of "start" to read the parameters of the body to the microcontroller. In addition, the mobile application retrieves 


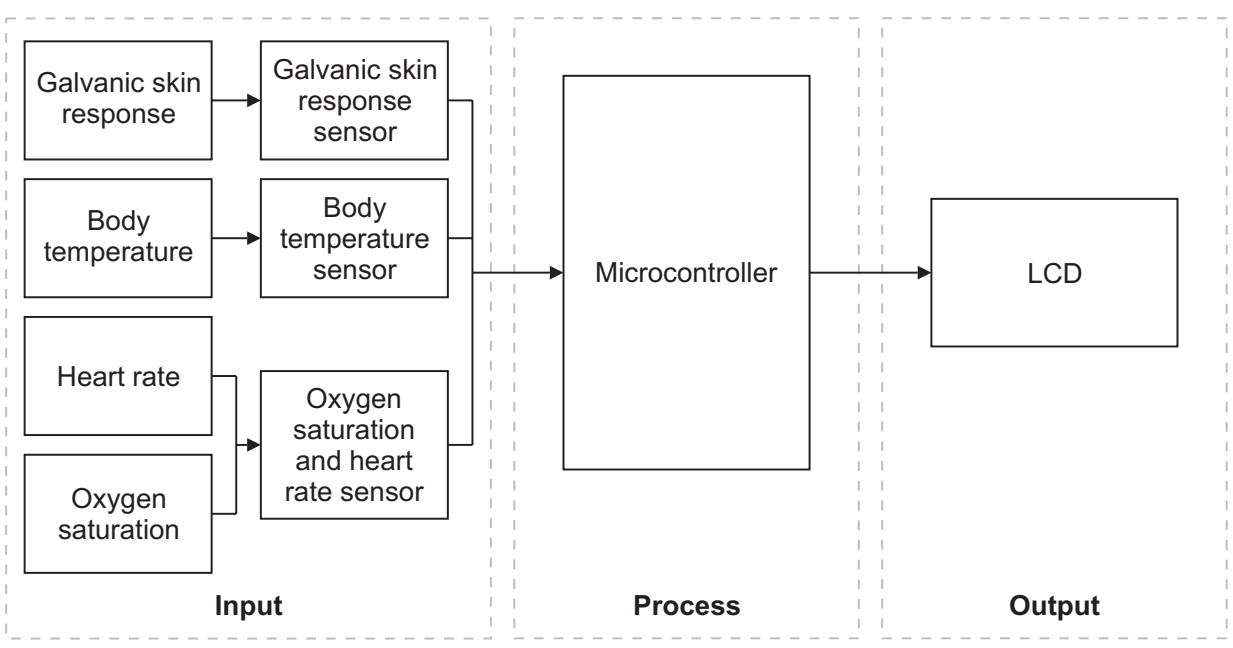

Figure 1. Block diagram of the system.

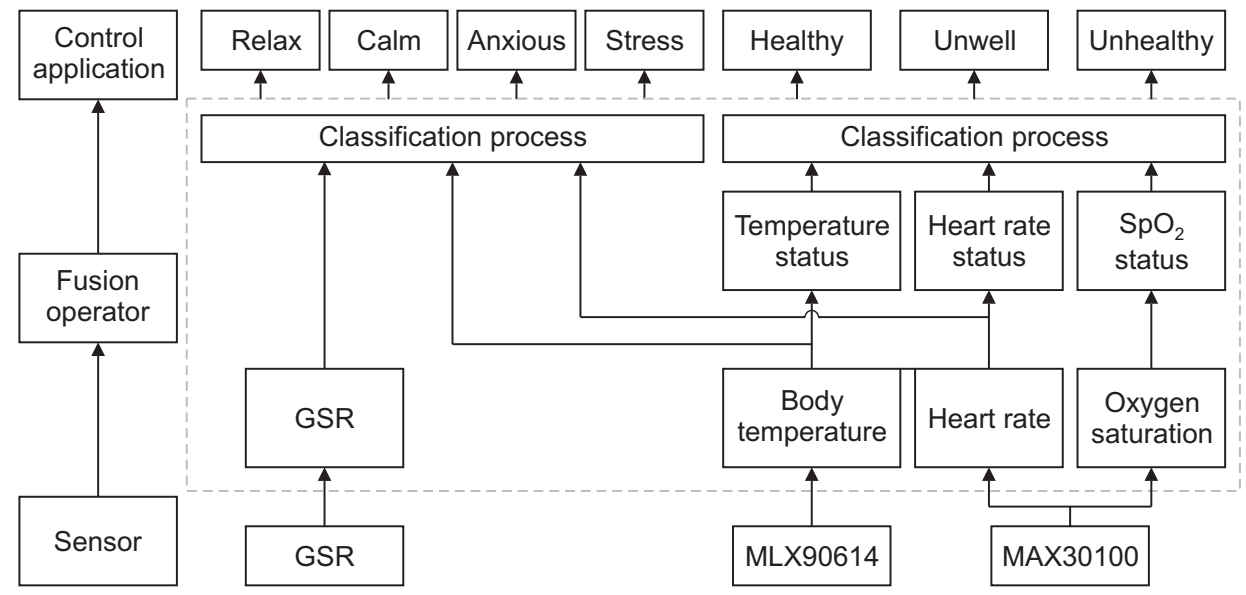

Figure 2. Fusion sensor design. GSR: galvanic skin response.

sensor reading data from the cloud database and determines the health status of the user's vital signs by using the IFTHEN algorithm and saves the measurement data into the data record page.

The work process of this device starts when the user presses the "start" sensor reading button in the mobile application, where the user must sign in first. If the button is pressed, the application will send a "start" instruction so that the device will start reading the user's biosignals for 25 seconds. The results of the biosignal reading process are processed by the microcontroller to determine the user's stress level. Then the biosignal data and the stress level are sent to the cloud database by the microcontroller to later be obtained by the mobile application. Data in the cloud database is then retrieved by the mobile application and processed using the IF-THEN algorithm to determine the health status of the user's vital signs. The data is then stored in the mobile application so that it can be used as a health data record.

\section{2) Fusion sensor design}

Referring to the software design of mobile applications and microcontrollers described earlier, the sensor fusion method is needed so that the device can make a final decision based on data collected by the sensor. The fusion sensor architecture designed for this device is shown in Figure 2.

The final decision on sensor fusion for stress levels and vital signs is made based on Kozier \& Erb's Fundamentals of Nursing (Tenth Edition), which is a basic manual for nursing [17]. The classification of vital signs is shown in Table 1.

In the implementation of the device, limits are required for body temperature, heart rate, and $\mathrm{SpO}_{2}$. The normal range of body temperature for young adults is considered to be between $36^{\circ} \mathrm{C}$ and $37.5^{\circ} \mathrm{C}\left(96.8^{\circ} \mathrm{F}\right.$ to $\left.99.5^{\circ} \mathrm{F}\right)$. There are two main changes in body temperature: pyrexia and hypothermia. Body temperature above the normal range is usually called pyrexia or hyperpyrexia. Pyrexia occurs when the body temperature ranges from $38^{\circ} \mathrm{C}\left(100.4^{\circ} \mathrm{F}\right)$ to $40^{\circ} \mathrm{C}$ $\left(104^{\circ} \mathrm{F}\right)$. A very high fever, such as $41^{\circ} \mathrm{C}\left(105.8^{\circ} \mathrm{F}\right)$, is called 
Table 1. Classification of vital sign final decisions

\begin{tabular}{llll}
\hline \multirow{2}{*}{\multicolumn{1}{c}{ Classification }} & \multicolumn{2}{c}{ User health status } \\
\cline { 2 - 4 } & Healthy & Unhealthy, with information & Not healthy, immediately contact a doctor \\
\hline Body temperature & Healthy & Fever, mild hypothermia & Hyperpyrexia, critical hypothermia \\
Heart rate & Healthy & Tachycardia, bradycardia & - \\
Oxygen saturation $\left(\mathrm{SpO}_{2}\right)$ & Healthy & Mild hypoxia & Critical hypoxia \\
\hline
\end{tabular}

Table 2. Classification of stress level final decisions

\begin{tabular}{lccc}
\hline Condition & GSR $(\mu \mathrm{S})$ & Heart rate $(\mathrm{bpm})$ & $\mathrm{H} \& \mathrm{~T}\left({ }^{\circ} \mathrm{C}\right)$ \\
\hline Relax & $<2$ & $60-70$ & $36-37$ \\
Calm & $2-4$ & $70-90$ & $35-36$ \\
Anxious & $4-6$ & $90-100$ & $33-35$ \\
Stress & $>6$ & $>100$ & $<33$ \\
\hline
\end{tabular}

GSR: galvanic skin response, H\&T: body temperature.

hyperpyrexia [17]. Body temperature below normal, less than $35^{\circ} \mathrm{C}\left(95^{\circ} \mathrm{F}\right)$, is known as hypothermia.

The normal heart rate range is 60 to 100 beats per minute (bpm). A fast heart rate in adults of more than $100 \mathrm{bpm}$ is called tachycardia, while a heart rate in adults less than 60 bpm is called bradycardia. If a client has tachycardia or bradycardia, then the client's apical pulse should be measured [17]. Factors that affect heart rate include age, gender, exercise, fever, medications, dehydration, stress, and pathology.

A healthy person with normal lungs and breathing air at sea level will have an arterial oxygen saturation of $95 \%$ to $100 \%$ [17]. If the oxygen saturation is $94 \%$ or lower, then the patient is suffering from hypoxia and needs to be treated quickly. If the saturation is less than $90 \%$, it can indicate a clinical emergency [18].

Based on Section 29 vital signs of the Fundamental of Nursing [17], stress affects changes in vital sign values. The final decision on the level of stress is based on other research as shown in Table 2 [19].

The algorithm used to determine the user's health status is IF-THEN, while the fuzzy logic algorithm is used to determine the stress level. The fuzzy logic block diagram for determining stress levels is shown in Figure 3.

The fuzzy membership functions of GSR, heart rate, body temperature, and stress level are shown in Figure 4, and the fuzzy associative memory (FAM) of fuzzy logic is shown in Table 3.

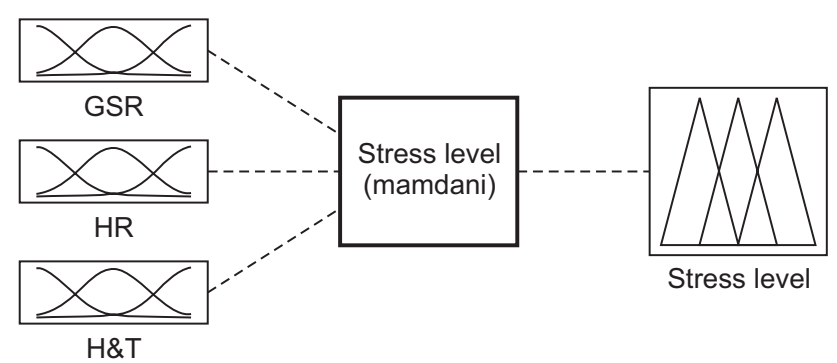

Figure 3. Fuzzy logic system design. GSR: galvanic skin response, HR: heart rate, H\&T: body temperature.

\section{Results}

\section{Sensors Testing}

The body temperature sensor was tested by comparing temperature readings from a heated measuring cup obtained by a mercury thermometer and the sensor used. The user of the proposed device can take his or her body temperature by placing a finger on the sensor with a distance of $\pm 4 \mathrm{~mm}$. Our test results showed that the temperature sensor prototype achieved a precision level of $99.926 \%$, an accuracy of $97.227 \%$, and a relative error of $0.271 \%$.

Heart rate sensor testing was done by comparing the results obtained by the prototype heart rate sensor with a comparison device, which was a heart rate sensor mounted on a smart phone that had been tested for accuracy. Heart rate data is obtained by the sensor when the user places a finger on the sensor, which has a red LED.

Oxygen saturation sensor testing was done by comparing the results obtained by prototype oxygen saturation sensor with a comparison device, which was an oxygen saturation sensor installed on a smart phone that had been tested for accuracy. The test results showed that the oxygen saturation sensor achieved a precision level of $99.7 \%$, an accuracy of $99.4 \%$, and a relative error of $0.3 \%$.

GSR sensor testing was done by comparing the resistance readings obtained by the sensor prototype with those obtained by a multimeter. The sensor prototype obtains readings from two electrodes attached to two fingers of one hand. The test results showed that the prototype GSR sensor 

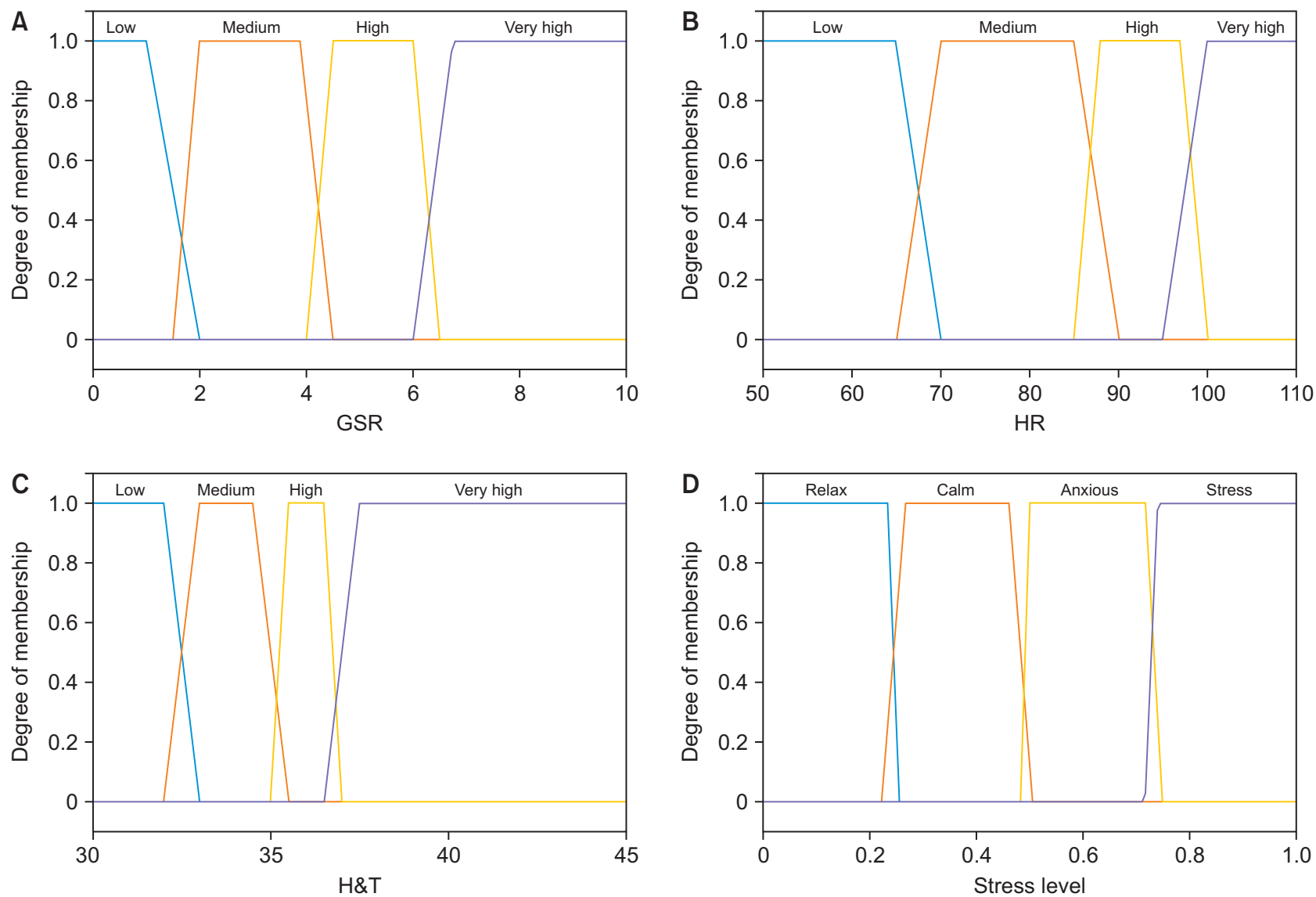

Figure 4. Membership function of (A) galvanic skin response (GSR), (B) heart rate (HR), (C) body temperature (H\&T), and (D) stress level.

Table 3. Fuzzy associative memory

\begin{tabular}{|c|c|c|c|c|c|}
\hline \multirow{2}{*}{ GSR } & \multirow{2}{*}{ Body temperature } & \multicolumn{4}{|c|}{ Heart rate } \\
\hline & & Low & Medium & High & Very high \\
\hline \multirow[t]{4}{*}{ Low } & Low & Relax & Anxious & Anxious & Anxious \\
\hline & Medium & Relax & Calm & Anxious & Anxious \\
\hline & High & Relax & Calm & Calm & Anxious \\
\hline & Very high & Relax & Relax & Calm & Anxious \\
\hline \multirow[t]{4}{*}{ Medium } & Low & Anxious & Anxious & Anxious & Stress \\
\hline & Medium & Calm & Calm & Anxious & Anxious \\
\hline & High & Calm & Calm & Calm & Anxious \\
\hline & Very high & Relax & Calm & Calm & Relax \\
\hline \multirow[t]{4}{*}{ High } & Low & Anxious & Calm & Anxious & Anxious \\
\hline & Medium & Anxious & Anxious & Anxious & Anxious \\
\hline & High & Calm & Calm & Anxious & Anxious \\
\hline & Very high & Calm & Calm & Anxious & Stress \\
\hline \multirow[t]{4}{*}{ Very high } & Low & Anxious & Anxious & Stress & Anxious \\
\hline & Medium & Anxious & Anxious & Anxious & Stress \\
\hline & High & Anxious & Calm & Anxious & Stress \\
\hline & Very high & Calm & Calm & Anxious & Stress \\
\hline
\end{tabular}

GSR: galvanic skin response. 
Table 4. Vital signs health status and stress level classification testing results

\begin{tabular}{|c|c|c|c|c|c|c|c|c|c|c|}
\hline \multirow[b]{2}{*}{ No } & \multicolumn{5}{|c|}{ Vital signs health status testing results } & \multicolumn{5}{|c|}{ Stress level classification testing results } \\
\hline & $\begin{array}{l}\text { Heart } \\
\text { rate } \\
(\mathrm{bpm})\end{array}$ & $\begin{array}{c}\text { Body } \\
\text { temperature } \\
\left({ }^{\circ} \mathrm{C}\right)\end{array}$ & $\begin{array}{c}\text { Oxygen } \\
\text { saturation } \\
(\%)\end{array}$ & Status & Information & $\begin{array}{l}\text { Heart rate } \\
\text { (bpm) }\end{array}$ & $\begin{array}{c}\text { Body } \\
\text { temperature } \\
\left({ }^{\circ} \mathrm{C}\right)\end{array}$ & $\begin{array}{l}\text { GSR } \\
(\mu S)\end{array}$ & Status & $\begin{array}{l}\text { Fuzzy } \\
\text { output }\end{array}$ \\
\hline 1 & 93.20 & 35.00 & 96 & Unhealthy & $\begin{array}{l}\text { Mild } \\
\text { hypothermia }\end{array}$ & 93.20 & 35.00 & 2.33 & Anxious & 0.61 \\
\hline 2 & 83.83 & 37.11 & 95 & Healthy & - & 83.83 & 37.11 & 2.60 & Calm & 0.36 \\
\hline 3 & 68.42 & 36.79 & 95 & Healthy & - & 68.42 & 36.79 & 2.25 & Calm & 0.26 \\
\hline 4 & 60.78 & 36.55 & 95 & Healthy & - & 60.78 & 36.55 & 1.92 & Calm & 0.34 \\
\hline 5 & 80.90 & 36.07 & 95 & Healthy & - & 80.90 & 36.07 & 2.22 & Calm & 0.36 \\
\hline 6 & 79.23 & 38.21 & 96 & Unhealthy & Fever/pyrexia & 79.23 & 38.21 & 2.14 & Calm & 0.36 \\
\hline 7 & 90.90 & 34.93 & 97 & Not healthy & Hypothermia & 90.90 & 34.93 & 6.00 & Anxious & 0.61 \\
\hline 8 & 82.00 & 37.00 & 94 & Unhealthy & Mild hypoxia & 82.00 & 37.00 & 2.97 & Calm & 0.36 \\
\hline 9 & 85.29 & 36.41 & 99 & Healthy & - & 85.29 & 36.41 & 0.77 & Calm & 0.36 \\
\hline 10 & 107.45 & 37.87 & 97 & Unhealthy & Tachycardia & 107.45 & 37.87 & 6.00 & Stress & 0.86 \\
\hline
\end{tabular}

GSR: galvanic skin response.
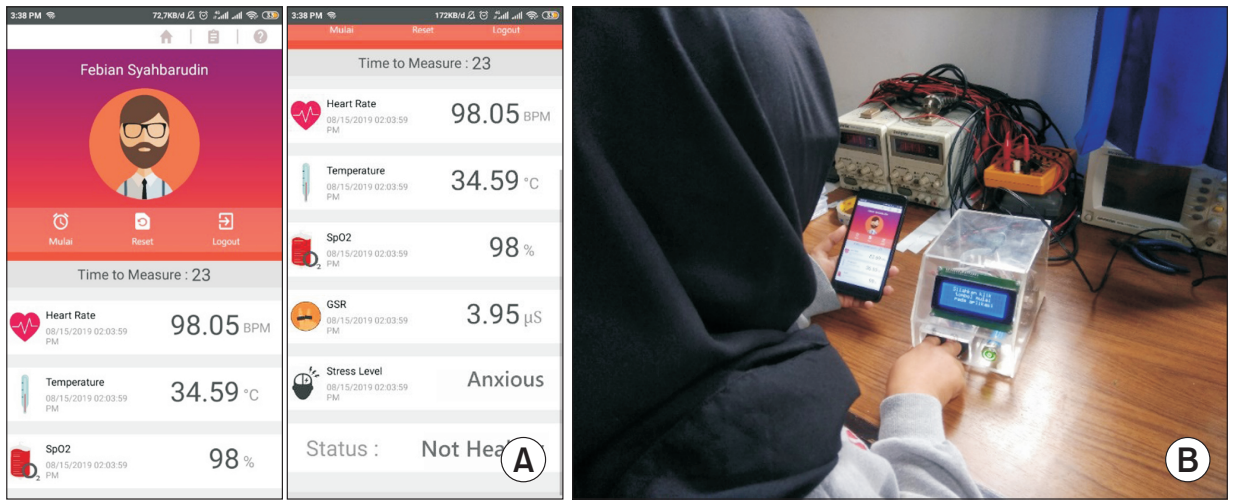

Figure 5. (A) Mobile application views and (B) intake of data from the body to the instrument.

Table 5. Results of overall fusion sensor testing

\begin{tabular}{|c|c|c|c|c|c|c|}
\hline \multirow{2}{*}{ No } & \multirow{2}{*}{$\begin{array}{l}\text { Heart rate } \\
\text { (bpm) }\end{array}$} & \multirow{2}{*}{$\begin{array}{c}\text { Body } \\
\text { temperature }\left({ }^{\circ} \mathrm{C}\right)\end{array}$} & \multirow{2}{*}{$\mathrm{GSR}(\mu \mathrm{S})$} & \multirow{2}{*}{$\begin{array}{c}\text { Oxygen } \\
\text { saturation (\%) }\end{array}$} & \multicolumn{2}{|c|}{ Status } \\
\hline & & & & & Stress level & Vital signs \\
\hline 1 & 87.17 & 37.03 & 1.64 & 97 & Calm & Healthy \\
\hline 2 & 81.01 & 37.39 & 4.65 & 99 & Calm & Healthy \\
\hline 3 & 74.01 & 36.51 & 4.49 & 100 & Calm & Healthy \\
\hline 4 & 84.98 & 36.05 & 3.04 & 100 & Calm & Healthy \\
\hline 5 & 102.92 & 35.95 & 1.58 & 99 & Anxious & Unhealthy \\
\hline 6 & 80.05 & 36.25 & 0.99 & 96 & Calm & Healthy \\
\hline 7 & 71.72 & 36.45 & 1.41 & 99 & Relax & Healthy \\
\hline 8 & 69.25 & 35.75 & 2.29 & 98 & Calm & Unhealthy \\
\hline 9 & 65.63 & 36.75 & 1.02 & 99 & Relax & Healthy \\
\hline 10 & 107.45 & 37.87 & 6.00 & 95 & Stress & Unhealthy \\
\hline
\end{tabular}

GSR: galvanic skin response. 
achieved a precision level of $97.402 \%$, an accuracy of $94.66 \%$, and a relative error of $2.59 \%$.

\section{Fusion Sensor Method for Vital Sign Health Status and Stress Level Testing}

The ultimate method of sensor fusion or the final output of this device is divided into two decisions, namely, the vital sign health status and stress level. The decision on the health status of vital signs is based on the data readings of heart rate, oxygen saturation, and body temperature. The health status of vital signs is determined using the IF-THEN algorithm. The health status of vital signs is presented as three possible outcomes: healthy, unhealthy (with information), and unhealthy (with advice to immediately contact a doctor). The ultimate method of sensor fusion (control application) or the final output of this device is divided into two decisions, namely, the health status of vital signs and stress levels. The stress level status decision determines the user's stress level based on the combined data readings of the heart rate, GSR, and body temperature. This determination of stress levels uses fuzzy logic algorithms. Stress level status is divided into four possible outcomes, namely, relaxed, calm, anxious, and stressed. Table 4 shows the results of vital sign health status and stress levels testing.

\section{Realization of Mobile Applications and Overall System Testing}

Human-machine interface (HMI) in the form of a mobile application on this device displays data from sensor readings and sensor fusion processing. This mobile application also saves the user's health record data. The mobile application was created using App Inventor, an open-source web application. In this application, Bluetooth is used to send commands to the microcontroller to "start" a sensor reading and "stop" a sensor reading. The Internet is used to receive sensor readings from the microcontroller and send/read data from the Firebase cloud database. The results obtained for the mobile application are shown in Figure 5A.

For overall system testing, the sensor fusion algorithm was tested as a whole to see whether the communication between the devices, databases, and mobile applications takes place properly or not. This testing was carried out to determine whether the proposed device can work in accordance with the expected functions and specifications.

The first test was the sensor fusion test as a whole. As shown in Figure 5B, data was retrieved from the user's body by attaching the user's index finger and middle finger of the right hand to the space located on the front of the prototype device. The results of the overall system test to determine the health status of vital signs and stress levels are shown in Table 5.

\section{Discussion}

A device to measure stress levels and vital signs based on sensor fusion was successfully designed and realized in accordance with the expected functions and specifications. The device can determine the stress level based on the vital signs read by sensors using the sensor fusion method. This device can also be connected to mobile applications via the Internet.

The sensors used in this device achieved relatively high levels of precision and accuracy. The precision levels achieved by the sensors used to obtain body temperature, oxygen saturation, and GSR readings were $99.926 \%, 99.7 \%$, and $99.302 \%$, respectively. The accuracy levels of body temperature, oxygen saturation, and GSR readings were $97.227 \%$, $99.4 \%$, and $98.6 \%$, respectively.

To display the stress level and vital sign status results, this device must be connected to the Internet. From the trial results, the minimum data transmission time is 1 second, while the average is \pm 3 to 8 seconds, depending on the speed of the Internet network used.

The classification algorithms developed in this study were not evaluated by consulting experts as a comparison. This research can be extended by consulting experts to verify the decisions made by the proposed device. In addition, the proposed device can be improved by utilizing additional sensors, such as microwave radar and thermography cameras.

\section{Conflict of Interest}

No potential conflict of interest relevant to this article was reported.

\section{ORCID}

Noor Cholis Basjaruddin (https://orcid.org/0000-0003-2003-7452) Febian Syahbarudin (https://orcid.org/0000-0003-1224-3205) Ediana Sutjiredjeki (https://orcid.org/0000-0001-5959-0867)

\section{References}

1. Yilmaz T, Foster R, Hao Y. Detecting vital signs with wearable wireless sensors. Sensors (Basel) 2010;10(12): 10837-62. 
2. Uthayakumar GS, Sivasubramanian A, Kisho AG. A novel technique to monitor human body vital signs. Int J Biomed Res 2013;4(09):642-59.

3. Khan F, Cho SH. A Detailed algorithm for vital sign monitoring of a stationary/non-stationary human through IR-UWB radar. Sensors (Basel) 2017;17(2):290.

4. Satwik K, Ramesh NV, Reshma SK. Estimation and monitoring of vital signs in the human body by using smart device. Int J Innov Technol Explor Eng 2019;8(5): 1035-8.

5. Sun G, Matsui T, Watai Y, Kim S, Kirimoto T, Suzuki S, et al. Vital-SCOPE: design and evaluation of a smart vital sign monitor for simultaneous measurement of pulse rate, respiratory rate, and body temperature for patient monitoring. J Sens 2018;2018:4371872.

6. Dionisi A, Marioli D, Sardini E, Serpelloni M. Autonomous wearable system for vital signs measurement with energy-harvesting module. IEEE Trans Instrum Meas 2016;65(6):1423-34.

7. American Psychological Association. Stress weakens the immune system [Internet]. Washington (DC): American Psychological Association; 2006 [cited at 2021 Jan 17]. Available from: https://www.apa.org/research/action/ immune.

8. Kupriyanov R, Zhdanov R. The eustress concept: problems and outlooks. World J Med Sci 2014;11(2):179-85.

9. Cho D, Ham J, Oh J, Park J, Kim S, Lee NK, Lee B. Detection of stress levels from biosignals measured in virtual reality environments using a kernel-based extreme learning machine. Sensors (Basel) 2017;17(10):2435.

10. Alberdi A, Aztiria A, Basarab A. Towards an automatic early stress recognition system for office environments based on multimodal measurements: a review. J Biomed Inform 2016;59:49-75.
11. Gjoreski M, Lustrek M, Gams M, Gjoreski H. Monitoring stress with a wrist device using context. J Biomed Inform 2017;73:159-70.

12. Akbar F, Mark G, Pavlidis I, Gutierrez-Osuna R. An empirical study comparing unobtrusive physiological sensors for stress detection in computer work. Sensors (Basel) 2019;19(17):3766.

13. Greene S, Thapliyal H, Caban-Holt A. A survey of affective computing for stress detection: evaluating technologies in stress detection for better health. IEEE Consum Electron Mag 2016;5(4):44-56.

14. Mozos OM, Sandulescu V, Andrews S, Ellis D, Bellotto N, Dobrescu R, et al. Stress detection using wearable physiological and sociometric sensors. Int J Neural Syst 2017;27(2):1650041.

15. Betti S, Lova RM, Rovini E, Acerbi G, Santarelli L, Cabiati $M$, et al. Evaluation of an integrated system of wearable physiological sensors for stress monitoring in working environments by using biological markers. IEEE Trans Biomed Eng 2018;65(8):1748-58.

16. Elmenreich W. Sensor fusion in time-triggered systems [dissertation]. Vienna, Austria: Vienna University of Technology; 2002.

17. Berman A, Snyder SJ, Frandsen G. Kozier \& Erb's fundamentals of nursing. 10th ed. Hoboken (NJ): Pearson; 2016.

18. World Health Organization. Pulse oximetry training manual. Geneva, Switzerland: World Health Organization; 2011.

19. Hadya PK. Rancang bangun alat pengukur tingkat stres menggunakan metode fuzzy logic [Design and development of stress level measurement using fuzzy logic method]. Surabaya, Indonesia: Institut Bisnis dan Informatika Stikom Surabaya; 2014. 